\title{
Clinical application of ultrasonography-guided percutaneous liver biopsy and its safety over 18 years
}

\author{
Young Chang ${ }^{1 *}$, Jun Il Kim², ${ }^{2 *}$ Bora Lee ${ }^{3}$, Sang Gyune Kim², Min Jung Jung ${ }^{4}$, Young Seok Kim², Soung Won Jeong', \\ Jae Young Jang ${ }^{1}$, and Jeong-Ju Yoo ${ }^{2}$
}

${ }^{1}$ Department of Internal Medicine, Soonchunhyang University College of Medicine, Seoul; ${ }^{2}$ Department of Internal Medicine, Soonchunhyang University College of Medicine, Bucheon; ${ }^{3}$ Department of Biostatistics, Chung-Ang University Graduate School, Seoul; ${ }^{4}$ Department of Pathology, Soonchunhyang University College of Medicine, Bucheon, Korea

\section{Graphical Abstract}

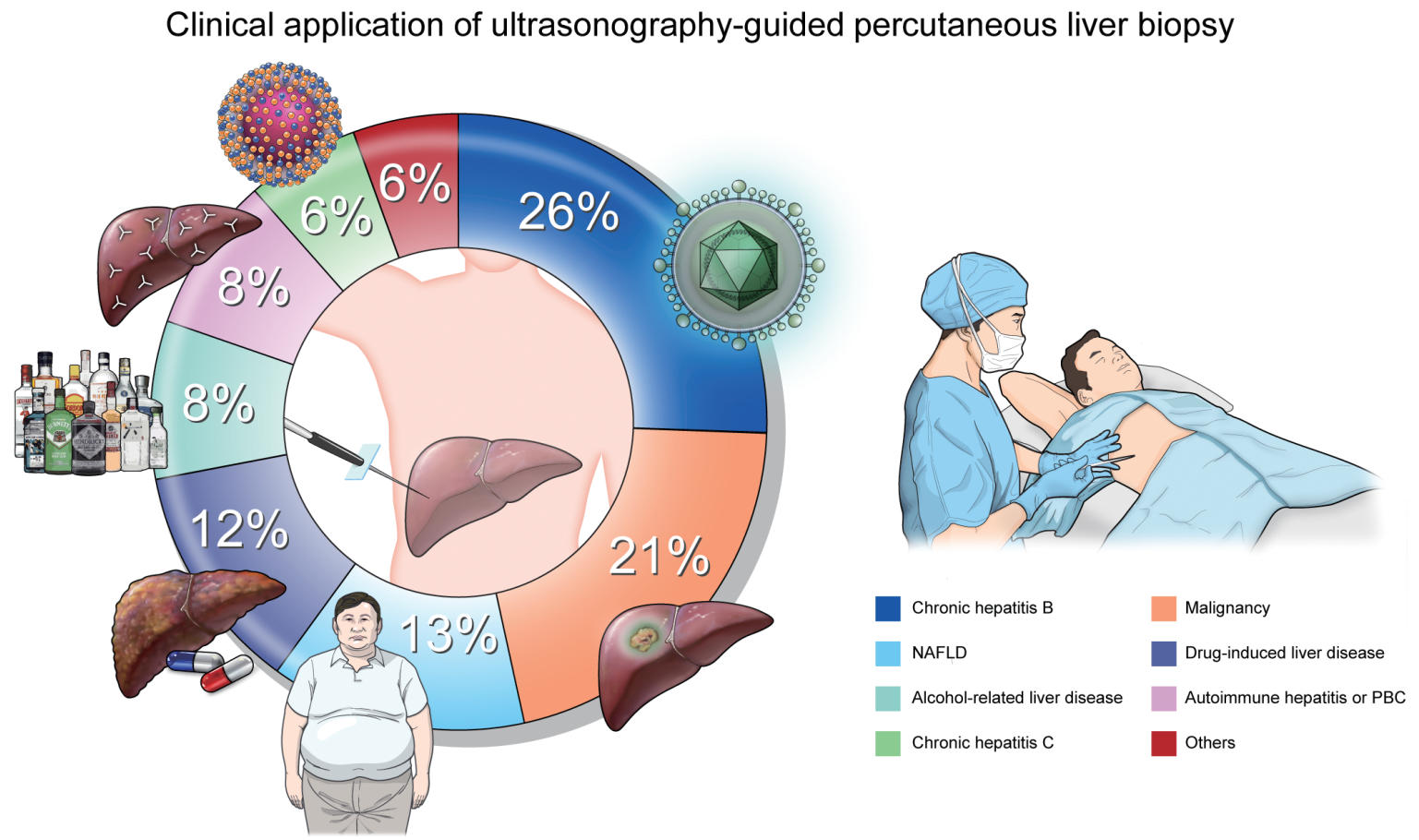

- A total of 1,944 patients underwent liver biopsies over the last 18 years.

- The overall rate of major adverse events was $0.05 \%$ (one case) and involved delayed bleeding at the biopsy site.

\section{Abbreviations:}

CT, computed tomography; DILI, drug-induced liver disease; HBV, hepatitis B virus; $\mathrm{HCC}$, hepatocellular carcinoma; MELD, model for end-stage liver disease; MRI, magnetic resonance imaging; NAFLD, nonalcoholic fatty liver disease; PBC, primary biliary cholangitis

*Y Chang and JI Kim contributed equally as co-first authors.
Corresponding author: Jeong-Ju Yoo

Division of Gastroenterology and Hepatology, Department of Internal Medicine, Soonchunhyang University Bucheon Hospital, 170 Jomaru-ro, Bucheon 14584, Korea

Tel: +82-32-621-5079, Fax: +82-32-621-5018

E-mail:puby17@naver.com

https://orcid.org/0000-0002-7802-0381 
Background/Aims: Liver biopsy (LB) remains the gold standard for the evaluation of liver disease. However, over the past two decades, many noninvasive tests have been developed and utilized in clinical practice as alternatives to LB. The aim of this study was to evaluate the clinical use and safety of $L B$ in the era of noninvasive assessment of liver fibrosis.

Methods: This retrospective study included 1,944 consecutive cases of LB performed between 2001 and 2018 in a tertiary hospital. All of the LBs were conducted under ultrasonography guidance with 18-gauge cutting needles.

Results: LBs were performed an average of approximately 108 times per year during the study period. Chronic hepatitis B (25.3\%) and suspected malignancy (20.5\%) were the two most common indications for LB. The use of LB for nonalcoholic fatty liver disease increased from $8.1 \%$ to $17.2 \%$ in the past 5 years compared to the last 10 years, while that for viral hepatitis decreased from $40.3 \%$ to $18.9 \%$. Discordance rate between the suspected diagnosis and the final diagnosis was $2.6 \%$ (51 cases). The overall rate of major adverse events was $0.05 \%$ (one case), which involved delayed bleeding at the biopsy site. Liver cirrhosis was observed in 563 cases (28.9\%), and the presence of cirrhosis did not affect the frequency of complications $(P=0.289)$.

Conclusions: LB is widely used in clinical practice as an irreplaceable diagnostic tool, even in the era of noninvasiveness. Ultrasonography-guided LB can be performed safely in patients with liver cirrhosis. (Clin Mol Hepatol 2020;26:318327)

Keywords: Liver biopsy; Indication; Complication

Liver biopsy has many clinical usefulness until recently, and when the procedure is performed using ultrasound, complications are low even in patients with cirrhosis.

\section{INTRODUCTION}

Percutaneous liver biopsy was first introduced in 1883. ' Since then, it has been the gold standard for the evaluation of liver disease. According to the American Association for the Study of Liver Disease guidelines, liver biopsy is an essential diagnostic tool for the diagnosis of parenchymal liver disease, and the evaluation of inflammation and fevers of unknown origin. ${ }^{2}$ However, liver biopsy is an invasive procedure associated with several complications. The most common complications were abdominal pain, which was reported in almost $30 \%$ of the patients, followed by bleeding $(0.3 \%)$ and death $(0.03 \%)$, respectively. ${ }^{3}$ However, these complication rates have been reported mainly in blind liver biopsies, and the complication rate is expected to decrease significantly under ultrasonography-guided liver biopsies. ${ }^{4}$ Currently, image-guided liver biopsies have replaced traditional blind liver biopsies. It is widely used to maximize the effectiveness of the procedure and minimize the complication rate. ${ }^{5}$

Recently, many noninvasive methods have been developed to replace liver biopsies. ${ }^{6}$ The most widely used noninvasive test is transient elastography. It has been used since 2005 to stage liver fibrosis and steatosis. ${ }^{7}$ Two-dimensional shear wave elastography can be used to determine the fibrosis stage of the liver based on the concurrent real-time grayscale. ${ }^{8}$ Moreover, magnetic resonance electrography can also be used to evaluate the entire liver fibrosis stage and is widely used to replace liver biopsies in clinical trials, especially phase II trials. ${ }^{9,10}$ Therefore, the role of a traditional liver biopsy is expected to change significantly in the immediate future.

In this study, we evaluated the changes in liver biopsy indications over the past 18 years and the safety of ultrasonographyguided liver biopsies in the era of noninvasive assessments of liver fibrosis.

\section{PATIENTS AND METHODS}

\section{Patients}

Between January 2001 and December 2018, we retrospectively collected data from patients undergoing ultrasonography-guided percutaneous liver biopsies at the Gastroenterology and Hepatology Department of a single tertiary center. A liver biopsy was performed when each investigator deemed it necessary to determine 
the cause and severity of the patient's liver disease. During this period, five patients underwent blind biopsies and 97 patients underwent computed tomography (CT)-guided biopsies. The 102 patients who underwent blind or CT-guided liver biopsies were excluded from the study. The final number of eligible patients included in the study was 1,944. The clinical indications for liver biopsy, the suspected diagnosis before the biopsy, and the final diagnosis after the biopsy were collected. The medications taken at the time of biopsy and complications after the biopsy were also reviewed. Liver cirrhosis was assessed by ultrasound, defined as the presence of a nodular liver surface, round edges, and hypoechoic nodules in the liver parenchyma, which represent regenerative nodules. ${ }^{11}$

The study protocol was approved by the Institutional Review Board of SoonChunHyang University Bucheon Hospital (IRB number SCHBC 2019-10-022). The study protocol conformed to the ethical guidelines of the World Medical Association Declaration of Helsinki.

\section{Liver biopsy}

Ultrasonography-guided liver biopsies were performed by four expert hepatologists experienced with more than 1,000 ultrasound procedures and over 200 liver biopsies. The patients were admitted to the hospital the day before the procedure and fasted for at least 12 hours. Prior to the procedure, routine laboratory tests, including coagulation parameters, chest X-rays, and abdominal ultrasounds were performed. During the procedure, a transthoracic approach was routinely used with the patient in the supine position. Local anesthesia using a $2 \%$ lidocaine solution was administered, and the biopsy was performed using an 18-gauge Tru-cut needle. After the liver biopsy, the standard compressive dressing was immediately applied to the wound and bed rest was advised for more than 6 hours for hemostasis. The patients remained under observation for one day following the procedure. The biopsy specimens were fixed in formalin and embedded in paraffin. Each biopsy specimen was analyzed by three experienced hospital pathologists. The histologic grading and staging of chronic hepatitis were performed based on standardized guidelines proposed by the Korean Study Group for the Pathology of Digestive Diseases. ${ }^{12}$

Table 1. Baseline characteristics of the study population

\begin{tabular}{|c|c|c|c|c|c|}
\hline & Total $(n=1,944)$ & Before $2008(n=456)$ & 2008 to $2012(n=859)$ & After 2013 ( $n=629)$ & $P$-value \\
\hline Age (years) & $48(37-57)$ & $47(35-57)$ & $46(37-54)$ & $51(41-60)$ & $<0.001$ \\
\hline Males & $1,135(58.4)$ & $336(73.7)$ & $481(56.0)$ & $318(50.5)$ & $<0.001$ \\
\hline \multicolumn{6}{|l|}{ Laboratory findings } \\
\hline Hemoglobin (g/dL) & $13.05 \pm 1.97$ & $13.26 \pm 2.02$ & $13.19 \pm 1.88$ & $12.7 \pm 2.02$ & $<0.001$ \\
\hline Platelets $\left(10^{3} / \mu \mathrm{L}\right)$ & $202.5 \pm 85.74$ & $193.78 \pm 85.23$ & $201.56 \pm 87.13$ & $210.1 \pm 83.64$ & 0.007 \\
\hline Total bilirubin (mg/dL) & $0.82(0.59-1.26)$ & $0.78(0.55-1.19)$ & $0.85(0.62-1.27)$ & $0.82(0.6-1.31)$ & 0.011 \\
\hline Albumin (g/dL) & $3.92 \pm 0.56$ & $3.93 \pm 0.53$ & $4.01 \pm 0.54$ & $3.79 \pm 0.58$ & $<0.001$ \\
\hline AST (U/L) & $51(31-94)$ & $52(31-98)$ & $48(30-91)$ & $52(32-95)$ & 0.195 \\
\hline $\operatorname{ALT}(\mathrm{U} / \mathrm{L})$ & $48(24-110)$ & $45(25-93.5)$ & $47(24-108.5)$ & $50(24-118.75)$ & 0.701 \\
\hline Creatinine (mg/dL) & $1.02 \pm 0.97$ & $1.05 \pm 0.83$ & $1.00 \pm 0.89$ & $1.02 \pm 1.15$ & 0.576 \\
\hline PT (INR) & $1.08 \pm 0.16$ & $1.09 \pm 0.14$ & $1.08 \pm 0.19$ & $1.09 \pm 0.15$ & 0.560 \\
\hline Liver cirrhosis & $563(28.96)$ & 141 (30.92) & $244(28.41)$ & $178(28.30)$ & 0.567 \\
\hline \multicolumn{6}{|l|}{ Medication } \\
\hline Antiplatelet & $56(2.88)$ & $9(1.97)$ & $32(3.73)$ & $15(2.38)$ & 0.129 \\
\hline Warfarin & $2(0.10)$ & $1(0.22)$ & $0(0.00)$ & $1(0.16)$ & 0.432 \\
\hline NSAID & $73(3.76)$ & $16(3.51)$ & $35(4.07)$ & $22(3.50)$ & 0.803 \\
\hline
\end{tabular}

Values are presented as mean \pm standard deviation, median (range) for continuous variables, or frequency (percentage) for categorical variables. The proportions are presented as percentages for categorical variables.

AST, aspartate aminotransferase; ALT, alanine aminotransferase; PT, prothrombin time; INR, international normalized ratio; NSAID, non-steroidal antiinflammatory drugs. 


\section{Statistical analysis}

Frequencies and percentages were used for the descriptive statistics. Statistical differences between the groups were investigated using the chi-squared test and Student's $t$-test for the comparison of two independent groups and ANOVA tests were used for groups of three or more. Spearman's analysis was used to investigate the correlations between the variables. All statistical analyses were performed using $R$ (version 3.3.3; The R Foundation for Statistical Computing, Vienna, Austria) and SPSS software (version 25.0; SPSS Inc., Chicago, IL, USA). Statistical significance was defined at $P<0.05$.

\section{RESULTS}

\section{Baseline characteristics of the study population}

Table 1 presents the baseline characteristics of the study population according to the year of the liver biopsy. A total of 1,944 patients underwent liver biopsies over the last 18 years. The median age of the study population was 48 years and $58 \%$ was male. The median age of the study population and the proportion of females tended to increase significantly over time. Despite advising patients to abstain from antiplatelet agents or anticoagu- lants, nine patients (1.97\%) continued taking antiplatelet agents, and one patient was undergoing warfarin treatment. These patients underwent biopsies after discontinuing the drug for 3 days after hospitalization.

\section{Changes in liver biopsy indications over time}

In all 1994 patients, hepatitis B virus (HBV) was the most common indication for a liver biopsy (25.3\%), followed by suspected malignancy (20.5\%), nonalcoholic fatty liver disease (NAFLD) (13.0\%), drug-induced liver disease (DILI) (11.7\%), and alcohol-related liver disease (8.2\%) during the overall study period (Fig. 1). The top three major indications for a liver biopsy were consistently chronic hepatitis $B$, malignancy, and NAFLD, but the priority of the indications changed over time (Table 2). Before 2008, malignancy $(34.6 \%)$ and chronic HBV (26.5\%) accounted for more than $50 \%$ of the liver biopsy indications. From 2008 to 2012, the most common indication was chronic HBV (31.3\%), followed by malignancy (15.7\%) and NAFLD (12.6\%). In the 5 years since 2013, the proportion of each of the three main indications was similar, with NAFLD accounting for the highest proportion, at $17.2 \%$.

Figure 2 shows that liver biopsies for chronic hepatitis B and C increased by 2012 , accounting for $40.3 \%$ of the total. In the last 5 years since 2013 , the proportion of viral hepatitis decreased substantially to $18.9 \%$. Liver biopsies to distinguish malignancies

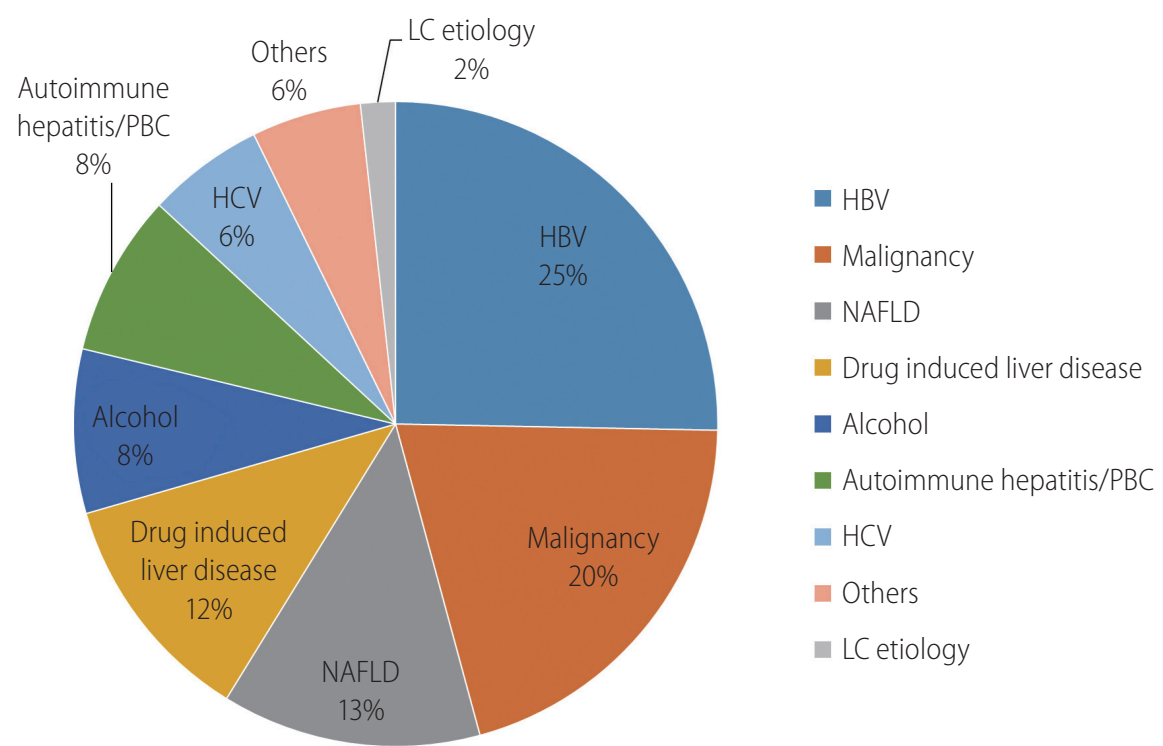

Figure 1. Indications for percutaneous liver biopsies during the overall study period (2001 to 2018). Chronic hepatitis B was the most common indication for liver biopsy, followed by malignancy, non-alcoholic fatty liver disease, drug-induced liver disease, and alcohol-related liver disease (8.2\%). LC, liver cirrhosis; HBV, hepatitis B virus; NAFLD, non-alcoholic fatty liver disease; PBC, primary biliary cholangitis; HCV, hepatitis C virus. 


\section{CLINCAL An MOLECULA
HEPATOLOGY}

Volume_26 Number_3 July 2020

also declined since 2008 , from $34.6 \%$ to $16.7 \%$. In contrast, liver biopsies for NAFLD and DILI increased gradually from $8.1 \%$ to $17.2 \%$ and $5.5 \%$ to $14.9 \%$, respectively, since 2008 . The proportion of biopsies for alcohol-related liver disease and autoimmune hepatitis/primary biliary cholangitis (PBC) did not change until 2012. However, since 2013, they have increased sharply from $5.9 \%$ to $12.2 \%$ and $5.4 \%$ to $13.8 \%$, respectively.

\section{Discordances in diagnoses before and after liver biopsies}

Table 3 shows the diagnostic agreement before and after biopsy. There were 51 cases (2.6\%) of discordance between the suspected diagnosis and the final diagnosis. The 51 discordant cases included 19 cases of suspected malignancies and 17 cases of suspected DILI. Of the 19 patients with suspected malignancies, 10

Table 2. Trends in the indications for liver biopsy over time

\begin{tabular}{|c|c|c|c|c|c|}
\hline & Total $(n=1,944)$ & Before $2008(n=456)$ & 2008 to $2012(n=859)$ & After $2013(n=629)$ & P-value \\
\hline HBV & $492(25.3)$ & $121(26.5)$ & $269(31.3)$ & $102(16.2)$ & $<0.001$ \\
\hline Malignancy & $398(20.5)$ & $158(34.6)$ & $135(15.7)$ & $105(16.7)$ & $<0.001$ \\
\hline NAFLD & $253(13.0)$ & $37(8.1)$ & $108(12.6)$ & $108(17.2)$ & $<0.001$ \\
\hline Drug-induced liver disease & $228(11.7)$ & $25(5.5)$ & $109(12.7)$ & $94(14.9)$ & $<0.001$ \\
\hline Autoimmune hepatitis or PBC & $158(8.1)$ & $25(5.5)$ & $46(5.4)$ & $87(13.8)$ & $<0.001$ \\
\hline $\mathrm{HCV}$ & $114(5.9)$ & $20(4.4)$ & $77(9.0)$ & $17(2.7)$ & $<0.001$ \\
\hline Others & $107(5.5)$ & $27(5.9)$ & $54(6.3)$ & $26(4.1)$ & $<0.001$ \\
\hline Etiology of liver cirrhosis & $34(1.7)$ & $11(2.4)$ & $10(1.2)$ & $13(2.1)$ & $<0.001$ \\
\hline
\end{tabular}

Values are presented as number (\%).

HBV, hepatitis B virus; NAFLD, nonalcoholic fatty liver disease; PBC, primary biliary cholangitis; $H C V$, hepatitis C virus.

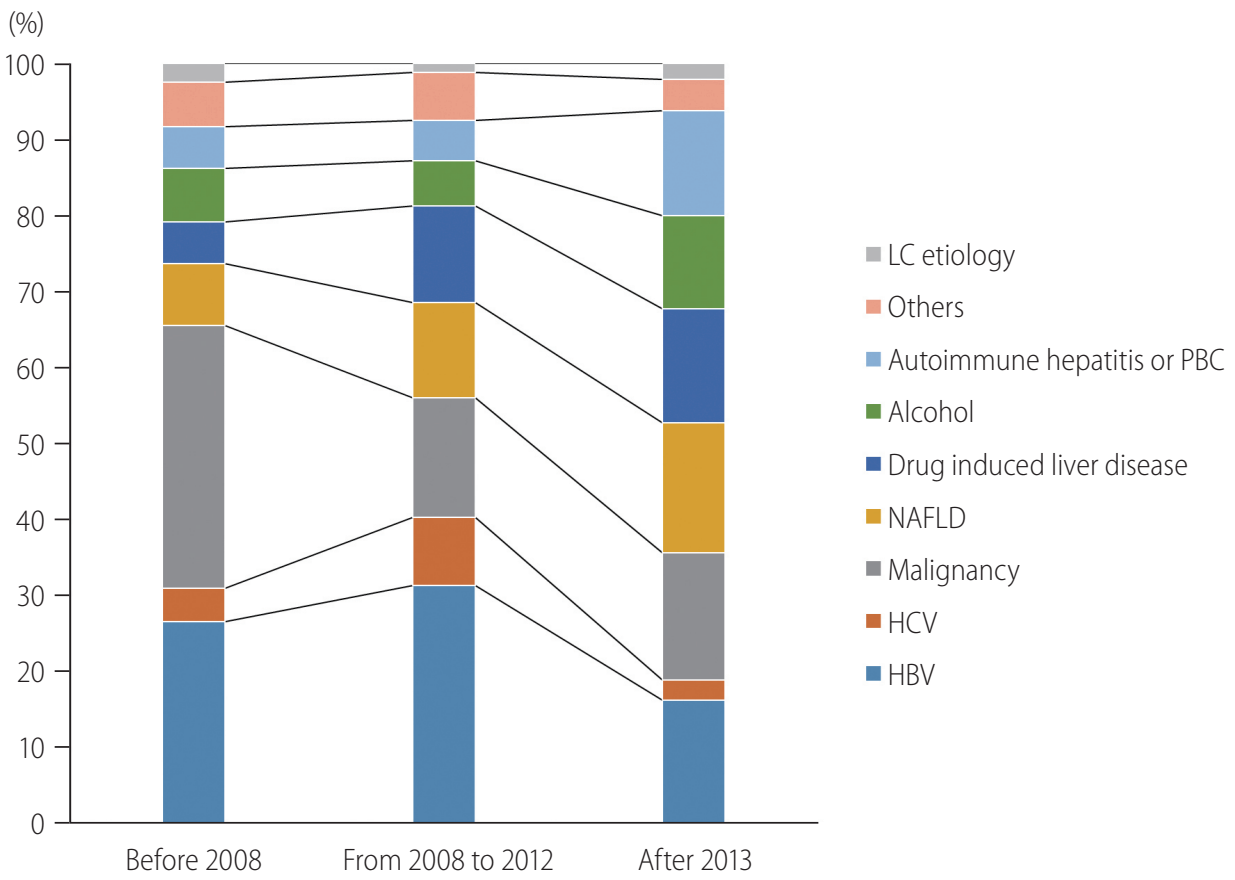

Figure 2. Changes in liver biopsy indications over time. Liver biopsies for viral hepatitis and malignancy decreased; whereas liver biopsies for non-alcoholic fatty liver disease, drug-induced liver disease, alcohol, and autoimmune hepatitis increased. LC, liver cirrhosis; PBC, primary biliary cholangitis; NAFLD, non-alcoholic fatty liver disease; HCV, hepatitis C virus; HBV, hepatitis B virus. 
were finally diagnosed as benign masses, such as hemangioma $(52.6 \%)$, and the remaining nine were diagnosed with other diseases. Of the 17 patients suspected with DILI, 11 (64.7\%) were finally diagnosed as autoimmune hepatitis, PBC or overlap syndrome, and five (29.4\%) as NAFLD. However, eight patients suspected of autoimmune hepatitis or PBC proved to be NAFLD or DILI after the liver biopsies.

In addition, there were two noticeable discordant cases. A 58-year-old male was suspected to have liver cirrhosis but it was difficult to identify whether the cause of the cirrhosis was HBV or alcohol by serological and radiological testing. However, after the liver biopsy, the pathologic analysis indicated that the main cause of the chronic liver injury was alcohol rather than HBV. Valuable information was obtained from the liver biopsy, and without the liver biopsy, it would not have been possible to accurately diagnose the patient using only his history or laboratory results. The second noticeable discordant case was a 48-year-old female diagnosed with a benign mass, although the suspected diagnosis was focal fat-sparing before the liver biopsy. She had a hypoechoic le- sion in the background of fatty liver by ultrasonography and was suspected to have a fatty liver with a focal fat-sparing zone. Additional CT and magnetic resonance imaging (MRI) tests could not differentiate between the focal fat-sparing zone vs. the benign mass in aspects such as focal nodular hyperplasia. A targeted liver biopsy was performed for the differential diagnosis of the focal lesion and it was finally confirmed as a hemangioma.

\section{Liver biopsy in cirrhosis}

Among the 1,944 patients included in the study population, $563(30.0 \%)$ had liver cirrhosis at the time of their liver biopsy. In these patients, a liver biopsy was conducted to determine the etiology of the liver cirrhosis and accurately determine the degree of liver fibrosis. Most of the pre-biopsy diagnoses were consistent with the final diagnoses, including cryptogenic liver cirrhosis. However, in contrast, the histological results of these patients revealed that $16 \%$ (90 of 563) had no liver cirrhosis. The histological results of the patients diagnosed with cirrhosis by ultrasound

Table 3. Diagnostic concordance before and after biopsy

\begin{tabular}{|c|c|c|c|c|c|c|c|c|c|c|}
\hline \multirow{2}{*}{ Final diagnosis } & \multicolumn{9}{|c|}{ Suspected diagnosis before liver biopsy } & \multirow{2}{*}{ Total } \\
\hline & HBV & $\mathrm{HCV}$ & Alcohol & NAFLD & $\mathrm{Al} / \mathrm{PBC}$ & DILI & Malignancy & LC & Etc. & \\
\hline Normal & & & & & & & 1 & & 35 & 36 \\
\hline HBV & 491 & & & & & & 3 & & & 494 \\
\hline $\mathrm{HCV}$ & & 113 & & & & & & & & 113 \\
\hline Alcohol & 1 & & 160 & & & & 1 & & 1 & 163 \\
\hline NAFLD & & & & 245 & 3 & 5 & 1 & & & 254 \\
\hline Autoimmune hepatitis & & & & 1 & 56 & 7 & & & 2 & 66 \\
\hline $\mathrm{PBC}$ & & & & & 55 & 2 & & 1 & & 58 \\
\hline Overlap syndrome & & & & & 24 & 2 & & & & 26 \\
\hline DILI & & 1 & & 1 & 5 & 200 & & 1 & 2 & 210 \\
\hline $\mathrm{HCC}$ & & & & & & & 278 & & & 278 \\
\hline IHCC & & & & & & & 53 & & & 53 \\
\hline Other cancer & & & & & & & 40 & & 1 & 41 \\
\hline Benign mass & & & & 1 & & & 10 & & 4 & 15 \\
\hline Cryptogenic LC & & & & & & 1 & 3 & 32 & 2 & 38 \\
\hline Rejection & & & & & & & & & 3 & 3 \\
\hline Infection & & & & & & & & & 6 & 6 \\
\hline Unknown & & & & 5 & 15 & 11 & 8 & & 51 & 90 \\
\hline Total & 492 & 114 & 160 & 253 & 158 & 228 & 398 & 34 & 107 & 1,944 \\
\hline
\end{tabular}

HBV, hepatitis B virus; HCV, hepatitis C virus; NAFLD, non-alcoholic fatty liver disease; Al, autoimmune hepatitis; PBC, primary biliary cholangitis; DILI, druginduced liver injury; LC, liver cirrhosis; HCC, hepatocellular carcinoma; IHCC, intrahepatic cholangiocarcinoma. 
were: F1 (nine patients, 1.60\%), F2 (six patients, 1.07\%), F3 (75 patients, 13.32\%), and F4 (473 patients, 84.01\%). As shown in Table 4, the patients with cirrhosis were significantly older than those without cirrhosis (median age of 53 years vs. 45 years) and comprised a higher proportion of males (66.1\% vs. $55.2 \%$ ). Among the cirrhotic patients, $20.2 \%$ of the patients showed ascites. Four hundred thirty-nine patients (78.0\%) were Child-Pugh class A and 124 (20.7\%) were Child-Pugh class B or C. The median model for end-stage liver disease (MELD) score was 8.7. The presence of cirrhosis did not affect the frequency of complications $(P=0.289)$.

\section{Complications after liver biopsy}

Painkillers were prescribed during hospitalization for $116 \mathrm{pa}$ tients (6.0\%) because of pain and there was no record of hypotension or pneumothorax. However, this was a retrospective study and thus, these minor complications may have been underestimated.

The major complication rate was $0.05 \%$. A single major complication associated with a liver biopsy occurred during the study period. The patient was a 61-year-old female who had cryptogenic liver cirrhosis. She had thrombocytopenia $(71,000 / \mu \mathrm{L})$ and prolonged prothrombin time (15.5 seconds). Her baseline Child-Pugh score was 6, and her MELD score was 13 . The liver biopsy was done without immediate complications and the patient was discharged without procedure-related complications by abdominal ultrasonography. However, four days post-liver biopsy, the patient was re-admitted due to abdominal pain and delayed bleeding from the biopsy site was found. The patient's vital signs and laboratory findings, including hemoglobin, were stable and no procedure was required for hemostasis. The patient was discharged successfully after supportive care for 5 days.

Table 4. Baseline characteristics of the study population with or without a diagnosis of liver cirrhosis

\begin{tabular}{|c|c|c|c|c|}
\hline & Total $(n=1,944)$ & Liver cirrhosis $(-)(n=1,382)$ & Liver cirrhosis (+) $(n=562)$ & $P$-value \\
\hline Age (years) & $48(37-57)$ & $45(34-55)$ & $53(45-61)$ & $<0.001$ \\
\hline Males & $1,135(58.35)$ & $763(55.21)$ & $372(66.07)$ & $<0.001$ \\
\hline $\operatorname{WBC}\left(10^{3} / \mu \mathrm{L}\right)$ & $5,700(4,500-7,040)$ & $5,995(4,800-7,300)$ & $5,070(3,905-6,500)$ & $<0.001$ \\
\hline Hemoglobin (g/dL) & $13.05 \pm 1.97$ & $13.29 \pm 1.92$ & $12.46 \pm 1.99$ & $<0.001$ \\
\hline Platelets $\left(10^{3} / \mu \mathrm{L}\right)$ & $200(146-251)$ & $220(175-263)$ & $137(92-194)$ & $<0.001$ \\
\hline Total bilirubin (mg/dL) & $0.82(0.59-1.26)$ & $0.76(0.56-1.13)$ & $0.97(0.72-1.47)$ & $<0.001$ \\
\hline Albumin (g/dL) & $3.92 \pm 0.56$ & $4.03 \pm 0.51$ & $3.64 \pm 0.57$ & $<0.001$ \\
\hline $\mathrm{AST}(\mathrm{U} / \mathrm{L})$ & $51(31-94)$ & $51(29-100)$ & $50(34-86)$ & 0.537 \\
\hline ALT (U/L) & $48(24-110)$ & $58(26-147)$ & $34(21-57)$ & $<0.001$ \\
\hline Creatinine (mg/dL) & $1.02 \pm 0.97$ & $1.02 \pm 0.97$ & $1.03 \pm 0.97$ & 0.820 \\
\hline PT (INR) & $1.08 \pm 0.16$ & $1.05 \pm 0.14$ & $1.17 \pm 0.19$ & $<0.001$ \\
\hline Ascites & & & & $<0.001$ \\
\hline None & 1,784 (91.72) & 1,335 (96.6) & 449 (79.75) & \\
\hline Mild & $137(7.04)$ & $43(3.11)$ & $94(16.7)$ & \\
\hline Moderate to severe & $24(1.23)$ & $4(0.29)$ & $20(3.55)$ & \\
\hline \multicolumn{5}{|l|}{ Child-Pugh class } \\
\hline Class A & N/A & N/A & $413(73.36)$ & \\
\hline Class B or C & N/A & N/A & $150(26.64)$ & \\
\hline MELD & N/A & N/A & $8.75(7.6-10.59)$ & \\
\hline Complication & 1 & 0 & 1 & 0.289 \\
\hline
\end{tabular}

Values are presented as mean \pm standard deviation, median (range) for continuous variables, or frequency (percentage) for categorical variables. The proportions are presented as percentages for categorical variables.

WBC, white blood cell; AST, aspartate aminotransferase; ALT, alanine aminotransferase; PT, prothrombin time; INR, international normalized ratio; N/A, not applicable; MELD, model for end-stage liver disease. 
Young Chang, et al. Changing trends in liver biopsy and safety

\section{DISCUSSION}

In this study, we retrospectively analyzed all of the cases of ultrasonography-guided percutaneous liver biopsies for the past 18 years. Currently, percutaneous liver biopsy under ultrasonography guidance has been the most generally used method. Only when there were specific reasons, such as high bleeding tendency or large amounts of perihepatic ascites, were transjugular or CTguided liver biopsies performed instead of ultrasonography-guided percutaneous liver biopsies. We included only patients who underwent ultrasonography-guided percutaneous liver biopsies to exclude those extreme cases and analyze a homogenous and standardized liver biopsy study population. The number of liver biopsies decreased from 2013 to 2018 and the indications for liver biopsies also changed by the period. The most common indication for liver biopsy during the entire study period was chronic HBV, followed by malignancy, and NAFLD. However, since 2013, the rate of NAFLD has increased rapidly, making it the most common indication. The rate of liver biopsies for chronic HBV and malignancy has declined, whereas the incidence of DILI, alcohol-related liver disease, and autoimmune hepatitis/PBC has increased sharply in the recent 5 years. Overall, a major adverse event of delayed bleeding at the biopsy site was identified, which was resolved by conservative management.

The decrease in liver biopsies for viral hepatitis was mainly attributed to the development of noninvasive methods for the assessment of liver fibrosis; the introduction of potent antiviral agents, such as entecavir, tenofovir, and direct-acting agents for hepatitis $C_{\text {; }}$ and the expansion of indications for antiviral treatment. In addition, advances in radiologic imaging techniques have reduced the number of liver biopsies for malignancies. Fatty liver has recently emerged as a major etiological factor underlying liver disease, which has rapidly increased the number of liver biopsies for NAFLD. These dynamic changes in indications for liver biopsy showed epidemiological changes over time in the study population.

Liver biopsy has been considered the most specific test to assess liver disease.' However, the clinical usefulness and impact of liver biopsies have been underestimated in the recent two decades due to the development of alternative noninvasive tests and new insight into the limitations of liver biopsies. ${ }^{13}$ Various types of liver elastography have been developed, with relatively accurate assessments of advanced liver fibrosis. The combination of medical history, serologic and radiologic evaluation, and liver elastography is adequate for the diagnosis of liver disease in most in- stances. ${ }^{14}$ In terms of solid liver lesions, multiphasic contrastenhanced CT or MRI can discern focal liver lesions in most cases. With the development of imaging techniques, a liver biopsy is rarely needed to distinguish focal liver lesions.

Liver biopsies, however, have their own unique role in the differential diagnosis and management of various liver diseases. A liver biopsy is essential for the diagnosis of autoimmune hepatitis, PBC — especially anti-mitochondrial antibody-negative — and several rare infiltrative diseases, such as amyloidosis and the hepatic involvement of lymphoma. ${ }^{13}$ In addition, only liver biopsy can differentiate simple steatosis from steatohepatitis in current clinical practice, which is important in treatment decisions in specific cases of chronic HBV infections. ${ }^{15,16}$ For the diagnosis of malignant tumors including hepatocellular carcinoma (HCC), histological confirmation is the most specific and useful method, especially when imaging findings are ambiguous. In fact, a retrospective analysis of the UNOS/OPTN database reported that $20.9 \%$ of the 789 patients who underwent liver transplantation for HCC were finally diagnosed with benign nodules. ${ }^{17}$ In our study as well, a considerable discrepancy $(2.6 \%)$ existed between the suspected diagnosis before liver biopsy and the final diagnosis. These results suggest the clinical importance of liver biopsies despite the development of noninvasive tests. In addition, among 563 patients who had ultrasonographic liver cirrhosis in this study, $16 \%$ were revealed not to have histologic liver cirrhosis. The ability to differentially diagnose the presence of liver cirrhosis and assess the exact fibrosis stage, as well as determine the etiology of chronic liver disease, is critical in several situations, especially to establish disease-specific treatments, such as antiviral therapy in viral hepatitis and steroid therapy in alcohol-related liver disease. Liver biopsies complement noninvasive methods, playing a critical role in the evaluation and management of liver fibrosis. ${ }^{10,18}$

In terms of safety, our study reported a substantially lower rate of major complications ( $0.05 \%)$ than previous reports. In addition, we found that liver biopsies were safe in patients with cirrhosis and/or ascites, contrary to the concerns of clinicians. Some studies have reported that perihepatic ascites did not significantly affect the major and minor complication rates of image-guided percutaneous liver biopsies. ${ }^{19,20}$ In this sense, most guidelines recommend total paracentesis before the biopsy, rather than prohibiting percutaneous liver biopsies in patients with tense ascites. ${ }^{21}$ We think that the reason for the low complication rate was mainly due to the use of ultrasonography.

There were several limitations associated with this study mainly due to its retrospective format. Minor complications, such as pain, 
could not be fully investigated because of the retrospective design. Therefore, the incidence of complications may be underestimated. Second, since this study was conducted in a single institution, it may not represent all patient types. However, some generalizations are possible because several doctors had performed liver biopsies independently even in a single institution and we examined all patients who underwent liver biopsies in the institution over 18 years, achieving a large study population. To represent the dynamic changes in liver biopsy indications in South Korea, further multicenter studies to validate our results are necessary.

In conclusion, a liver biopsy is an irreplaceable diagnostic method, even in this era of noninvasive techniques. Also, we found that ultrasonography-guided liver biopsy was safe for patients with liver cirrhosis.

\section{Authors' contribution}

Conceptualization: JJ Yoo; methodology: Y Chang and JI Kim; formal analysis: B Lee; investigation: JI Kim; resources: SW Jeong and JJ Jang; data curation: SG Kim; writing - original draft preparation, Y Chang and JJ Yoo; writing - review \& editing, JJ Yoo; visualization, B Lee; and supervision, SG Kim and YS Kim.

\section{Acknowledgements}

This work was supported by Soonchunhyang University research fund.

\section{Conflicts of Interest}

The authors have no conflicts to disclose.

\section{REFERENCES}

1. Bravo AA, Sheth SG, Chopra S. Liver biopsy. N Engl J Med 2001;344:495-500.

2. Rockey DC, Caldwell SH, Goodman ZD, Nelson RC, Smith AD; American Association for the Study of Liver Diseases. Liver biopsy. Hepatology 2009;49:1017-1044.

3. Cadranel JF, Nousbaum JB. Current trends in liver biopsy indications in chronic liver diseases. Presse Med 2012;41:1064-1070.

4. Szymczak A, Simon K, Inglot M, Gladysz A. Safety and effectiveness of blind percutaneous liver biopsy: analysis of 1412 procedures. Hepat Mon 2012;12:32-37.

5. Parente FVC, Moura EA, Santos JAMD, Lima MVA. US-guided percutaneous core liver biopsy: analysis of 171 cases from a single oncology service. Arq Gastroenterol 2018;55:208-211.
6. Lurie Y, Webb M, Cytter-Kuint R, Shteingart S, Lederkremer GZ. Non-invasive diagnosis of liver fibrosis and cirrhosis. World J Gastroenterol 2015;21:11567-11583.

7. Tapper EB, Castera L, Afdhal NH. FibroScan (vibration-controlled transient elastography): where does it stand in the United States practice. Clin Gastroenterol Hepatol 2015;13:27-36.

8. Kim DW, Suh CH, Kim KW, Pyo J, Park C, Jung SC. Technical performance of two-dimensional shear wave elastography for measuring liver stiffness: a systematic review and meta-analysis. Korean J Radiol 2019;20:880-893.

9. Singh S, Venkatesh SK, Wang Z, Miller FH, Motosugi U, Low RN, et al. Diagnostic performance of magnetic resonance elastography in staging liver fibrosis: a systematic review and meta-analysis of individual participant data. Clin Gastroenterol Hepatol 2015;13:440451.e6.

10. Yoo JJ, Kim W, Kim MY, Jun DW, Kim SG, Yeon JE, et al. Recent research trends and updates on nonalcoholic fatty liver disease. Clin Mol Hepatol 2019;25:1-11.

11. Matsutani S, Fukuzawa T, Ebara M, Ohto M. Ultrasonography in the diagnosis of liver cirrhosis. Nihon Rinsho 1994;52:56-62.

12. Yu E; Korean Study Group for the Pathology of Digestive Diseases. Histologic grading and staging of chronic hepatitis: on the basis of standardized guideline proposed by the Korean Study Group for the Pathology of Digestive Diseases. Taehan Kan Hakhoe Chi 2003:9:42-46.

13. Tapper EB, Lok AS. Use of liver imaging and biopsy in clinical practice. N Engl J Med 2017;377:756-768.

14. Tapper EB, Saini SD, Sengupta N. Extensive testing or focused testing of patients with elevated liver enzymes. J Hepatol 2017;66:313319.

15. European Association for the Study of the Liver (EASL); European Association for the Study of Diabetes (EASD); European Association for the Study of Obesity (EASO). EASL-EASD-EASO clinical practice guidelines for the management of non-alcoholic fatty liver disease. J Hepatol 2016;64:1388-1402.

16. European Association for the Study of the Liver. EASL 2017 clinical practice guidelines on the management of hepatitis $B$ virus infection. J Hepatol 2017;67:370-398.

17. Freeman RB, Mithoefer $A$, Ruthazer R, Nguyen $K$, Schore A, Harper $A$, et al. Optimizing staging for hepatocellular carcinoma before liver transplantation: a retrospective analysis of the UNOS/OPTN database. Liver Transpl 2006;12:1504-1511.

18. Lo RC, Kim H. Histopathological evaluation of liver fibrosis and cirrhosis regression. Clin Mol Hepatol 2017;23:302-307.

19. Murphy FB, Barefield KP, Steinberg HV, Bernardino ME. CT- or sonography-guided biopsy of the liver in the presence of ascites: frequency of complications. AJR Am J Roentgenol 1988;151:485486. 
Young Chang, et al. Changing trends in liver biopsy and safety

20. Little AF, Ferris JV, Dodd GD 3rd, Baron RL. Image-guided percutaneous hepatic biopsy: effect of ascites on the complication rate. Radiology 1996;199:79-83.
21. Grant A, Neuberger J. Guidelines on the use of liver biopsy in clinical practice. British Society of Gastroenterology. Gut 1999;45 Suppl 4:IV1-IV11. 